\title{
A generalization of a theorem by Kato on Navier-Stokes equations
}

\section{Marco Cannone}

Abstract. We generalize a classical result of T. Kato on the existence of global solutions to the Navier-Stokes system in $C\left([0, \infty) ; L^{3}\left(\mathbb{R}^{3}\right)\right)$. More precisely, we show that if the initial data are sufficiently oscillating, in a suitable Besov space, then Kato's solution exists globally. As a corollary to this result, we obtain a theorem on existence of self-similar solutions for the Navier-Stokes equations.

\section{Introduction.}

In the study of the Cauchy problem for the Navier-Stokes equations governing the time evolution of the velocity $v(t, x)$ and the pressure $p(t, x)$ of an incompressible viscous fluid filling all of $\mathbb{R}^{3}$

$$
\left\{\begin{array}{l}
\frac{\partial v}{\partial t}-\Delta v=-(v \cdot \nabla) v-\nabla p \\
\nabla \cdot v=0 \\
v(0)=v_{0}
\end{array}\right.
$$

there is considerable interest in finding global solutions $v(t, x)$ which are strongly continuous from the interval $[0, \infty)$ and take values in an abstract Banach space, whose norm is invariant under the transformation $f(\cdot) \longmapsto \lambda f(\lambda \cdot)$, for all $\lambda>0$. 
Following [1], we will call such a space a limit space for the study of the Navier-Stokes equations. A typical example is given by the Lebesgue space $L^{3}\left(\mathbb{R}^{3}\right)[2],[3]$, but one can also consider the homogeneous Sobolev space $\dot{H}^{1 / 2}\left(\mathbb{R}^{3}\right)[4]$, [5], or the homogeneous MorreyCampanato space $\dot{M}_{2}^{3}\left(\mathbb{R}^{3}\right)$ [6]-[9], or more sophisticated and somewhat esoteric examples as the Besov or Triebel-Lizorkin spaces [1].

The reason why these limit spaces arise naturally in the study of the Navier-Stokes equations is very simple. Suppose that $v(t, x)$ and $p(t, x)$ solve the system

$$
\left\{\begin{array}{l}
\frac{\partial v}{\partial t}-\Delta v=-(v \cdot \nabla) v-\nabla p \\
\nabla \cdot v=0
\end{array}\right.
$$

then, the same holds true for $v_{\lambda}=\lambda v\left(\lambda^{2} t, \lambda x\right)$ and $p_{\lambda}=\lambda^{2} p\left(\lambda^{2} t, \lambda x\right)$ for any positive $\lambda$.

In other words, these spaces have, as far as the space variable is concerned, the same scaling invariance as the Navier-Stokes equations, so that it appears very natural to solve (0.1) when the initial data $v_{0}$ (which depend on the $x$-variable only) belong to a Banach limit space.

In a previous article [10] (see also [1]), we gave a detailed analysis of the local well-posedness of the Cauchy problem (0.1) with initial data in an abstract Banach space. Although the algorithm we presented in [1], [10] allows one to recover - in a very abstract way - many previous known results on existence of local solutions for the system (0.1), it does not shed any light on the case of a limit Banach space.

In order to understand why and where the difficulties arise when dealing with such a limit space, let us briefly recall the standard procedure used in the study of the initial value problem (0.1).

The idea is the following. One first transforms the classical system (0.1) into the following mild integral equation

$$
v(t)=S(t) v_{0}+B(v, v)(t)
$$

where

$$
B(v, u)(t)=-\int_{0}^{t} \mathbb{P} S(t-s) \nabla \cdot(v \otimes u)(s) d s
$$

$\mathbb{P}$ and $S$ being respectively the projection onto divergence free vector fields and the heat semigroup. 
Then, it is customary to obtain the existence and uniqueness of a strongly continuous global $(T=\infty)$ or local $(T<\infty)$ solution $v(t, x) \in$ $C([0, T) ; X)$ of $(0.3), X$ being an abstract Banach space, by means of the standard contraction algorithm. Of course, the main difficulty in applying such an algorithm is to establish, a priori, the bicontinuity of the bilinear operator $B(v, u)(t)$ in $C([0, T) ; X) \times C([0, T) ; X) \longrightarrow$ $C([0, T) ; X)$.

In the case of the Lebesgue space $L^{p}\left(\mathbb{R}^{3}\right)$, a straightforward application of Young inequality implies that

$$
\begin{aligned}
& \sup _{0 \leq t<T}\|B(v, u)(t)\|_{p} \\
& \quad \leq C\left(\int_{0}^{T} t^{-1 / 2-3 /(2 p)}\right) \sup _{0 \leq t<T}\|v(t)\|_{p} \sup _{0 \leq t<T}\|u(t)\|_{p}
\end{aligned}
$$

thus showing that $B(v, u)(t)$ is bicontinuous in $C\left([0, T) ; L^{p}\left(\mathbb{R}^{3}\right)\right)$ as long as $p>3$.

On the other hand, it is not known whether or not the bilinear operator $B(v, u)$ is continuous in the limit $L^{3}\left(\mathbb{R}^{3}\right)$ setting. What is certainly true is that, even if the bilinear operator $B(v, u)(t)$ turns out not to be bicontinuous in such a limit space $L^{3}\left(\mathbb{R}^{3}\right)$, say in $C\left([0, T) ; L^{3}\left(\mathbb{R}^{3}\right)\right)$, this would not necessarily imply a nonexistence theorem of mild solutions $v(t, x) \in C\left([0, T) ; L^{3}\left(\mathbb{R}^{3}\right)\right)$ for the Navier-Stokes equations.

The problem of solving the integral equation $(0.3)$ in the $L^{3}\left(\mathbb{R}^{3}\right)$ setting was first tackled in 1984 by $T$. Kato [2], who was able to circumvent the problem of the possible noncontinuity of $B(v, u)(t)$ in $C\left([0, T) ; L^{3}\left(\mathbb{R}^{3}\right)\right)$ (i.e. the nonintegrability at the origin of the function $t^{-1}$ appearing in $(0.5)$ for $\left.p=3\right)$.

Kato's masterstroke was to remark that, in order to obtain an existence theorem in $C\left([0, T) ; L^{3}\left(\mathbb{R}^{3}\right)\right)$ for the Navier-Stokes equations, it is sufficient to show that the bilinear operator $B(v, u)(t)$ is continuous in a suitable subspace of $C\left([0, T) ; L^{3}\left(\mathbb{R}^{3}\right)\right)$. This subspace $K$ is made up of the functions $v(t, x) \in C\left([0, T) ; L^{3}\left(\mathbb{R}^{3}\right)\right)$ such that, moreover,

$$
t^{\alpha / 2} v(t, x) \in C\left([0, T) ; L^{q}\left(\mathbb{R}^{3}\right)\right)
$$

and

$$
\lim _{t \rightarrow 0} t^{\alpha / 2}\|v(t)\|_{q}=0
$$

and normed by

$$
\||v|\|_{K}=\sup _{0 \leq t<T}\|v(t)\|_{3}+\sup _{0 \leq t<T} t^{\alpha / 2}\|v(t)\|_{q}
$$


$q$ being a fixed constant satisfying $3<q \leq 6$ and $\alpha=\alpha(q)=1-3 / q$.

In other words, Kato's idea was to look for solutions in a space of vector-valued functions equipped with two norms: the first is the natural one, while the second controls the balance of the smoothing property of the heat semigroup $S(t)$ against the apparition of singularities by the quadratic term $B(v, v)(t)$.

Now, it is easy to observe that not only is the bilinear operator $B(v, u)(t)$ bicontinuous in this norm $\||\cdot|\|_{K}$, but also $S(t) v_{0} \in K$ as long as $v_{0} \in L^{3}\left(\mathbb{R}^{3}\right)$. All this is sufficient to deduce, by means of a standard contraction procedure, an existence theorem of global mild solutions for the Navier-Stokes equations in $C\left([0, \infty) ; L^{3}\left(\mathbb{R}^{3}\right)\right)$ and small initial data in $L^{3}\left(\mathbb{R}^{3}\right)$, the uniqueness of the solution being guaranteed only in $K$, and not, in general, in the natural space $C\left([0, \infty) ; L^{3}\left(\mathbb{R}^{3}\right)\right)$. More precisely, Kato's theorem (in a somewhat simplified version [1]) reads as follows [2]

Kato's Theorem. Let $q$ be fixed, $3<q \leq 6$, and $\alpha=\alpha(q)=1-3 / q$, then there exists an absolute constant $\delta>0$, such that if $v_{0}$ belongs to $L^{3}\left(\mathbb{R}^{3}\right),\left\|v_{0}\right\|_{3}<\delta$ and $\nabla \cdot v_{0}=0$ (in the distributional sense), then there exists a global mild solution of the Navier-Stokes equations in $C\left([0, \infty) ; L^{3}\left(\mathbb{R}^{3}\right)\right)$. Moreover, this solution is the only one such that

$$
\begin{gathered}
v(t, x) \in C\left([0, \infty) ; L^{3}\left(\mathbb{R}^{3}\right)\right), \\
t^{\alpha / 2} v(t, x) \in C\left([0, \infty) ; L^{q}\left(\mathbb{R}^{3}\right)\right)
\end{gathered}
$$

and

$$
\lim _{t \rightarrow 0} t^{\alpha / 2}\|v(t)\|_{q}=0
$$

The aim of this paper is to prove that Kato's result holds true under a much weaker condition on the initial data. In order to make it clear, let us introduce (Definition 1.3) the Besov space $\dot{B}_{q}^{-\alpha, \infty}\left(\mathbb{R}^{3}\right)$, $q$ and $\alpha(q)$ being chosen as before. It is quite easy to prove that

$$
L^{3}\left(\mathbb{R}^{3}\right) \hookrightarrow \dot{B}_{q}^{-\alpha, \infty}\left(\mathbb{R}^{3}\right)
$$

but that these two spaces are different, for $|x|^{-1} \in \dot{B}_{q}^{-\alpha, \infty}\left(\mathbb{R}^{3}\right)$ and $|x|^{-1} \notin L^{3}\left(\mathbb{R}^{3}\right)$ (Lemma 1.2). 
Now, if we observe that for any tempered distribution $v_{0} \in \mathcal{S}^{\prime}\left(\mathbb{R}^{3}\right)$ the $\left\|v_{0}\right\|_{\dot{B}_{q}^{-\alpha, \infty}}$ norm is equivalent to $\sup _{t \geq 0} t^{\alpha / 2}\left\|S(t) v_{0}\right\|_{q}($ Lemma 1.1), then Kato's global solution exists and satisfies (0.9)-(0.11) under the weaker conditions: $v_{0} \in L^{3}\left(\mathbb{R}^{3}\right),\left\|v_{0}\right\|_{\dot{B}_{q}^{-\alpha, \infty}}<\delta$ and $\nabla \cdot v_{0}=0$ in the sense of distributions (Theorem 1.1).

Before delving into the details of this weak formulation of Kato's theorem, let us comment on the condition $\left\|v_{0}\right\|_{\dot{B}_{q}^{-\alpha, \infty}}<\delta$.

To fix the ideas, let us suppose that $v_{0}(x)$ is an arbitrary $L^{3}\left(\mathbb{R}^{3}\right)$ function and let $w_{k}(x)$ be a sequence of functions such that $\left\|w_{k}\right\|_{\infty} \leq c$ (uniformly in $k$ ) and that $w_{k} \rightarrow 0$ in the sense of distributions as $k$ goes to infinity.

In other words, let us suppose that the functions $w_{k}$ are uniformly bounded and are more and more oscillating as $k$ increases. Under these hypotheses, it is easy to prove that $w_{k}(x) v(x)$ tends to 0 in the strong topology of $\dot{B}_{q}^{-\alpha, \infty}\left(\mathbb{R}^{3}\right)$ (Lemma 2.1) in spite of the fact that if $\left|w_{k}(x)\right|=1$ (almost everywhere in $x$, for any $k$ ), then $\left\|w_{k} v_{0}\right\|_{3}=\left\|v_{0}\right\|_{3}$. A typical example of a sequence $w_{k}(x)$ fulfilling all these conditions is given by the exponential function, say $w_{k}(x)=\exp (i x \cdot k)$ (here, $k$ is a vector and we let $|k|$ go to $\infty)$.

The importance of the weaker condition, $\left\|v_{0}\right\|_{\dot{B}_{q}^{-\alpha, \infty}}<\delta$ instead of $\left\|v_{0}\right\|_{3}<\delta$, is now clear and can be formulated as follows: in order to prove the existence of Kato's global solution, all we need is sufficiently oscillating initial data. Of course, we have to pay attention to the divergence condition on the initial data for, in general, the divergence operator $\nabla$. does not commutate with the functions $w_{k}(x)$. Nevertheless, this does hold true asymptotically as $k$ goes to infinity (Lemma 2.2 ), which is exactly the situation we have to deal with.

Another remarkable property of the Besov spaces $\dot{B}_{q}^{-\alpha, \infty}\left(\mathbb{R}^{3}\right)$ is, as we pointed out before, that they contain among their elements homogeneous functions of degree -1 , such that e.g. $|x|^{-1}$. This is a crucial point when looking for solutions to the Navier-Stokes equations which satisfy the scaling property

$$
v(t, x)=v_{\lambda}(t, x)=\lambda v\left(\lambda^{2} t, \lambda x\right), \quad \text { for all } \lambda>0
$$

In fact, whenever they exist, these particular solutions $v(t, x)$, which are usually called self-similar solutions, are such that their initial value $v(0, x)$ is a homogeneous function of degree -1 . We will show in this paper how to obtain, by using the above mentioned weak formulation of Kato's theorem, an existence theorem of self-similar solutions $v(t, x)$ 
with initial data $v_{0}$ homogeneous of degree -1 , divergence-free, and sufficiently small in the Besov space $\dot{B}_{q}^{-\alpha, \infty}\left(\mathbb{R}^{3}\right)$ (Theorem 3.2).

The plan of the paper is the following. Section 1 contains the basic definitions and the proof of the main theorem. Section is devoted to illustrating that $\left\|v_{0}\right\|_{\dot{B}_{q}^{-\alpha, \infty}}<\delta$ is satisfied for sufficiently oscillating initial data $v_{0}$. Finally, sections 3 and 4 deal with the existence of self-similar solutions for the Navier-Stokes equations in $\dot{B}_{q}^{-\alpha, \infty}\left(\mathbb{R}^{3}\right)$.

\section{The proof of the main Theorem.}

We study the Cauchy problem for the Navier-Stokes equations governing the time evolution of the velocity

$$
v(t, x)=\left(v_{1}(t, x), v_{2}(t, x), v_{3}(t, x)\right)
$$

and the pressure $p(t, x)$ of an incompressible fluid filling all of $\mathbb{R}^{3}$

$$
\left\{\begin{array}{l}
\frac{\partial v}{\partial t}-\Delta v=-(v \cdot \nabla) v-\nabla p \\
\nabla \cdot v=0 \\
v(0)=v_{0} .
\end{array}\right.
$$

We will focus our attention on the existence of global solutions to (1.1) in $C\left([0, \infty) ; L^{3}\left(\mathbb{R}^{3}\right)\right)$, the space of continuous functions $v(t, x)$ of $t \in$ $[0, \infty)$ with values in the Banach space $L^{3}\left(\mathbb{R}^{3}\right)$ of vector distributions.

Here and in the following, we say that a vector $a=\left(a_{1}, a_{2}, a_{3}\right)$ belongs to a function space $X$ if $a_{j} \in X$ holds for every $j=1,2$ and 3 , and we put $\|a\|=\max _{1 \leq j \leq 3}\left\|a_{j}\right\|$.

Before stating the main hypotheses concerning the initial data $v_{0}$ under which the system $(1.1)$ will be solved in $C\left([0, \infty) ; L^{3}\left(\mathbb{R}^{3}\right)\right)$, let us recall some definitions, which will be useful in the sequel.

\subsection{The operator $\mathbb{P}$.}

We let $\partial_{j}=-i \partial / \partial x_{j},\left(i^{2}=-1\right)$ and we indicate by $R_{j}=$ $\partial_{j}(-\Delta)^{-1 / 2}$, for $j=1,2$ and 3 , the Riesz transformation.

For an arbitrary vector field $v(x)=\left(v_{1}(x), v_{2}(x), v_{3}(x)\right)$ on $\mathbb{R}^{3}$, we set

$$
z(x)=\sum_{j=1}^{3}\left(R_{j} v_{j}\right)(x)
$$


and finally we define the operator $\mathbb{P}$ by

$$
(\mathbb{P} v)_{k}(x)=v_{k}(x)-\left(R_{k} z\right)(x), \quad 1 \leq k \leq 3 .
$$

$\mathbb{P}$ is a pseudo-differential operator of degree zero and is an orthogonal projection onto the kernel of the divergence operator.

Making use of this projection operator $\mathbb{P}$ and the heat semigroup $S(t)=\exp (t \Delta)$, it is now a straightforward procedure to reduce the classical partial differential system (1.1) into the mild integral equation

$$
v(t)=S(t) v_{0}-\int \mathbb{P} S(t-s) \nabla \cdot(v \otimes v)(s) d s
$$

Accordingly, a solution of the equation (1.4) will be called a mild solution of the Navier-Stokes equations. It is not difficult to see that a mild solution of the Navier-Stokes (1.4) is actually a classical solution of the system (1.1) (and conversely). It would be inappropriate and beyond the scope of this paper to present a proof of this equivalence here. Fore more details on the subject, we refer the reader to [3], [4].

\subsection{The Littlewood-Paley decomposition.}

Let us choose a real rotation invariant function $\varphi$ in the Schwartz space $\mathcal{S}\left(\mathbb{R}^{3}\right)$ whose Fourier transform is such that

$$
0 \leq \hat{\varphi}(\xi) \leq 1, \quad \begin{cases}\hat{\varphi}(\xi)=1, & \text { if }|\xi| \leq \frac{3}{4} \\ \hat{\varphi}(\xi)=0, & \text { if }|\xi| \geq \frac{3}{2}\end{cases}
$$

and let

$$
\begin{gathered}
\psi(x)=8 \varphi(2 x)-\varphi(x), \\
\varphi_{j}(x)=2^{3 j} \varphi\left(2^{j} x\right), \quad j \in \mathbb{Z}, \\
\psi_{j}(x)=2^{3 j} \psi\left(2^{j} x\right), \quad j \in \mathbb{Z} .
\end{gathered}
$$

We denote by $S_{j}$ and $\Delta_{j}$, respectively, the convolution operators with $\varphi_{j}$ and $\psi_{j}$. Finally, the set $\left\{S_{j}, \Delta_{j}\right\}_{j \in \mathbb{Z}}$ (actually a set) is the Littlewood-Paley decomposition of the unity, say

$$
I=S_{0}+\sum_{j \geq 0} \Delta_{j}=\sum_{j \in \mathbb{Z}} \Delta_{j} .
$$


It is worthwhile to recall that only the first of the two series, say $f=S_{0} f+\sum_{j \geq 0} \Delta_{j} f$, applies without any restriction on the tempered distribution $f$. On the other hand, the identity $f=\sum_{j \in \mathbb{Z}} \Delta_{j} f$ is to be understood modulo polynomials (see [11] for a complete and general discussion on the matter).

\subsection{The Besov spaces $\dot{B}_{q}^{-\alpha, \infty}\left(\mathbb{R}^{3}\right)$.}

Let $q$ be fixed in $1 \leq q \leq \infty$ and $\alpha \in \mathbb{R}$. A tempered distribution $v \in \mathcal{S}^{\prime}\left(\mathbb{R}^{3}\right)$ belongs to the Besov space $\dot{B}_{q}^{-\alpha, \infty}\left(\mathbb{R}^{3}\right)$ if and only if the following norm

$$
\|v\|_{\dot{B}_{q}^{-\alpha, \infty}}=\sup _{j \in \mathbb{Z}} 2^{-j \alpha}\left\|\Delta_{j} v\right\|_{q}
$$

is finite. Here, $\Delta_{j}$ is, for any $j \in \mathbb{Z}$, the convolution operator with the function $\psi_{j}$ given in a Littlewood-Paley decomposition of unity.

The following lemma will provide a different characterization of the Besov space $\dot{B}_{q}^{-\alpha, \infty}\left(\mathbb{R}^{3}\right)$ in terms of the heat semigroup and will be one of the staple ingredients of the proof of Theorem 1.1.

Lemma 1.1. Let $q$ be fixed in $1 \leq q \leq \infty$ and $\alpha>0$. For any tempered distribution $v \in \mathcal{S}^{\prime}\left(\mathbb{R}^{3}\right)$, the following four norms

$$
\begin{aligned}
& \sup _{j \in \mathbb{Z}} 2^{-j \alpha}\left\|\Delta_{j} v\right\|_{q}, \\
& \sup _{j \in \mathbb{Z}} 2^{-j \alpha}\left\|S_{j} v\right\|_{q}, \\
& \sup _{t \geq 0} t^{\alpha / 2}\|S(t) v\|_{q}, \\
& \sup _{t \geq 0}\|S(t) v\|_{\dot{B}_{q}^{-\alpha, \infty}},
\end{aligned}
$$

are equivalent, and will be referred to in the sequel by $\|v\|_{\dot{B}_{q}^{-\alpha, \infty}}$.

The first equivalence (1.11) if and only if (1.12) is easy to prove if we recall that $\Delta_{j}=S_{j+1}-S_{j}$ for all $j \in \mathbb{Z}$, that $S_{j+1}=\sum_{k \leq j} \Delta_{k}$ and that $\alpha>0$. Of course, when passing from (1.11) to (1.12) the proviso stated after (1.9), on the equivalence modulo the polynomials, is still required. 
Let us examine the equivalence (1.12) if and only if (1.13). In order to see this, it is sufficient to observe that for $t=4^{-j}$ the convolution operator $S(t)$ essentially reduces to the operator $S_{j}$. Of course, this is the case because the Fourier transform of $S(t)$ is given by $\exp \left(-t|\xi|^{2}\right)$ which has essentially the same properties stated in (1.5). The equivalence (1.12) if and only if (1.13) can now be shown using the same techniques as in the proof of the independence of the particular choice of $\varphi$ in the Littlewood-Paley decomposition (see [12] for a proof).

The next step is the equivalence (1.13) if and only if (1.14). Here the proof is evident and left to the reader.

Lemma 1.2. Let $q_{1}$ and $q_{2}$ be two fixed constants in $3 \leq q_{1} \leq q_{2} \leq \infty$ and put $\alpha_{1}=1-3 / q_{1}$ and $\alpha_{2}=1-3 / q_{2}$. We have the following chain of continuous imbeddings

$$
L^{3}\left(\mathbb{R}^{3}\right) \hookrightarrow \dot{B}_{q_{1}}^{-\alpha_{1}, \infty}\left(\mathbb{R}^{3}\right) \hookrightarrow \dot{B}_{q_{2}}^{-\alpha_{2}, \infty}\left(\mathbb{R}^{3}\right) .
$$

This result is a consequence of the Bernstein's inequalities [13] which allow us to deduce, for any $j \in \mathbb{Z}, 3 \leq q_{1} \leq q_{2} \leq \infty$ and any tempered distribution $v$, the following chain of inequalities

$$
2^{-j \alpha_{2}}\left\|\Delta_{j} v\right\|_{q_{2}} \leq c 2^{-j \alpha_{1}}\left\|\Delta_{j} v\right\|_{q_{1}} \leq c\left\|\Delta_{j} v\right\|_{3} \leq c\|v\|_{3},
$$

which finally implies (1.15).

At this point, we would like to remark that the above inclusions are strict ones. For example, if we consider the function $|x|^{-1}$, here $x=\left(x_{1}, x_{2}, x_{3}\right)$, then $|x|^{-1} \in \dot{B}_{q}^{-\alpha, \infty}\left(\mathbb{R}^{3}\right)(q>3, \alpha=1-3 / q)$, in spite of the fact that $|x|^{-1} \notin L^{3}\left(\mathbb{R}^{3}\right)$.

In Section 4 we will give a complete characterization, i.e. a necessary and sufficient condition, of which homogeneous functions of degree -1 belong to the space $\dot{B}_{q}^{-\alpha, \infty}\left(\mathbb{R}^{3}\right)(q>3, \alpha=1-3 / q)$.

Here we limit ourselves to the case of the function $|x|^{-1}, x=$ $\left(x_{1}, x_{2}, x_{3}\right)$. More generally, we want to show that if the restriction to the unit sphere $S^{2}$ of a tempered distribution function $v$, homogeneous of degree -1 , belongs to $L^{\infty}\left(S^{2}\right)$, then $v$ belongs to $\dot{B}_{q}^{-\alpha, \infty}\left(\mathbb{R}^{3}\right)(q>3$, $\alpha=1-3 / q)$. In fact, thanks to the homogeneity of $v$, we have

$$
\begin{aligned}
\|v\|_{\dot{B}_{q}^{-\alpha, \infty}} & =\sup _{j \in \mathbb{Z}} 2^{-j \alpha}\left\|S_{j} v\right\|_{q} \\
& =\sup _{j \in \mathbb{Z}} 2^{-j(\alpha-1+3 / q)}\left\|S_{0}\right\|_{q} \\
& =\left\|S_{0}\right\|_{q} .
\end{aligned}
$$


Now, if $\left.v\right|_{S^{2}} \in L^{\infty}\left(S^{2}\right)$, we have

$$
\left|S_{0} v\right| \leq \frac{C}{1+|x|}
$$

which finally gives $v \in \dot{B}_{q}^{-\alpha, \infty}\left(\mathbb{R}^{3}\right)$ as long as $q>3$. This concludes our remark.

We are now in a position to generalize Kato's result. To this end let us first introduce the Banach space $G$ which is made up by functions $v(t, x)$ satisfying

$$
\begin{gathered}
v(t, x) \in C\left([0, \infty) ; L^{3}\left(\mathbb{R}^{3}\right)\right), \\
t^{\alpha / 2} v(t, x) \in C\left([0, \infty) ; L^{q}\left(\mathbb{R}^{3}\right)\right)
\end{gathered}
$$

and

$$
\lim _{t \rightarrow 0} t^{\alpha / 2}\|v(t)\|_{q}=0
$$

and normed by

$$
\|v\|_{G}=\sup _{t>0}\|v(t)\|_{\dot{B}_{q}^{-\alpha, \infty}}+\sup _{t>0} t^{\alpha / 2}\|v(t)\|_{q} .
$$

This definition makes sense because, as we noticed in Lemma 1.2, the Lebesgue space $L^{3}\left(\mathbb{R}^{3}\right)$ is continuously imbedded in $\dot{B}_{q}^{-\alpha, \infty}\left(\mathbb{R}^{3}\right)$.

The theorem that we will prove here is the following.

Theorem 1.1. Let $q$ be fixed in $3<q \leq 6$ and $\alpha=\alpha(q)=1-3 / q$, then there exists an absolute constant $\delta>0$ such that if $v_{0}$ belongs to $L^{3}\left(\mathbb{R}^{3}\right),\left\|v_{0}\right\|_{\dot{B}_{q}^{-\alpha, \infty}}<\delta$, and $\nabla \cdot v_{0}=0$ (in the sense of distributions), then there exists a global mild solution of the Navier-Stokes equations in $C\left([0, \infty) ; L^{3}\left(\mathbb{R}^{3}\right)\right)$. Moreover, this solution is the only one such that

$$
\begin{gathered}
v(t, x) \in C\left([0, \infty) ; L^{3}\left(\mathbb{R}^{3}\right)\right), \\
t^{\alpha / 2} v(t, x) \in C\left([0, \infty) ; L^{q}\left(\mathbb{R}^{3}\right)\right)
\end{gathered}
$$

and

$$
\lim _{t \rightarrow 0} t^{\alpha / 2}\|v(t)\|_{q}=0
$$


The proof of this Theorem 1.1 can be obtained easily by virtue of the following three lemmata.

Lemma 1.3. If $v_{0} \in L^{3}\left(\mathbb{R}^{3}\right)$, then $S(t) v_{0} \in G$.

First, by Lemma 1.1, the $G$ norm of $S(t) v_{0}$ is equivalent to the $\dot{B}_{q}^{-\alpha, \infty}\left(\mathbb{R}^{3}\right)$ norm of $v_{0}$. Next, as $v_{0}$ belongs to $L^{3}\left(\mathbb{R}^{3}\right)$ and as $L^{3}\left(\mathbb{R}^{3}\right)$ is a separable space, a straightforward application of the Banach-Steinhaus theorem shows that (1.19)-(1.21) hold with $v(t)$ replaced by $S(t) v_{0}$. This concludes the proof of the lemma.

Lemma 1.4. The bilinear operator $B(v, u)(t)$ defined by

$$
B(v, u)(t)=-\int_{0}^{t} \mathbb{P} S(t-s) \nabla \cdot(v \otimes u)(s) d s
$$

is bicontinuous in $G \times G \longrightarrow G$.

For the sake of simplicity, we will prove the lemma in its scalar version. More precisely, following [1], we will consider the scalar version of the bilinear operator $B(v, u)(t)$, given by

$$
B(f, g)(t)=-\int_{0}^{t}(t-s)^{-2} \Theta\left(\frac{\cdot}{\sqrt{t-s}}\right) *(f g)(s) d s,
$$

where $f=f(t, x)$ and $g=g(t, x)$ are two scalar fields in $G$ and $\Theta=$ $\Theta(x)$ is an analytic function of $x$ which is $O\left(|x|^{-4}\right)$ at infinity.

First of all, Young inequality (here the condition $q \leq 6$ appears) gives

$$
\|B(f, g)(t)\|_{3} \leq \int_{0}^{t}(t-s)^{-2}\left\|\theta\left(\frac{\cdot}{\sqrt{t-s}}\right)\right\|_{m}\|f(s) g(s)\|_{q / 2} d s,
$$

where

$$
\frac{1}{3}=\frac{1}{m}+\frac{2}{q}-1
$$

thus showing that

$$
\begin{aligned}
\|B(f, g)(t)\|_{3} \leq & \left(\int_{0}^{t}(t-s)^{-2+3 /(2 m)} s^{-\alpha} d s\right)\|\Theta\|_{m} \\
& \cdot\left(\sup _{t>0} t^{\alpha / 2}\|f(t)\|_{q}\right)\left(\sup _{t>0} t^{\alpha / 2}\|g(t)\|_{q}\right) \\
= & c\left(\sup _{t>0} t^{\alpha / 2}\|f(t)\|_{q}\right)\left(\sup _{t>0} t^{\alpha / 2}\|g(t)\|_{q}\right) .
\end{aligned}
$$


The next step is to evaluate the second term of the $G$-norm. To this end, we use again Young's inequality and find

$$
\|B(f, g)(t)\|_{q} \leq \int_{0}^{t}(t-s)^{-2}\left\|\theta\left(\frac{\cdot}{\sqrt{t-s}}\right)\right\|_{n}\|f(s) g(s)\|_{q / 2} d s
$$

where

$$
\frac{1}{q}=\frac{1}{n}+\frac{2}{q}-1
$$

this gives the desired result

$$
\begin{aligned}
\|B(f, g)(t)\|_{q} \leq & \left(\int_{0}^{t}(t-s)^{-2+3 /(2 n)} s^{-\alpha} d s\right)\|\Theta\|_{n} \\
& \cdot\left(\sup _{t>0} t^{\alpha / 2}\|f(t)\|_{q}\right)\left(\sup _{t>0} t^{\alpha / 2}\|g(t)\|_{q}\right) \\
= & c t^{-\alpha / 2}\left(\sup _{t>0} t^{\alpha / 2}\|f(t)\|_{q}\right)\left(\sup _{t>0} t^{\alpha / 2}\|g(t)\|_{q}\right) .
\end{aligned}
$$

Let us now check the validity of condition (1.21) for the bilinear term $B(v, u)(t)$. Actually, we will prove a more precise statement.

In fact, not only is

$$
\lim _{t \rightarrow 0} t^{\alpha / 2}\|B(f, g)(t)\|_{q}=0,
$$

whenever

$$
\lim _{t \rightarrow 0} t^{\alpha / 2}\|f(t)\|_{q}=\lim _{t \rightarrow 0} t^{\alpha / 2}\|g(t)\|_{q}=0
$$

but also, if the latter condition is fulfilled, we have

$$
\lim _{t \rightarrow 0}\|B(f, g)(t)\|_{3}=0
$$

In particular, this last property is very important in the proof of Theorem 1.1, because it guarantees that any solution $v(t, x) \in G$ of the integral equation (1.4) with data $v_{0}$ in $L^{3}\left(\mathbb{R}^{3}\right)$ tends to $v_{0}$ in the strong topology of $L^{3}\left(\mathbb{R}^{3}\right)$ and is unique in $G$.

Let us now verify condition (1.36). This is trivial because, if

$$
s^{\alpha / 2}\|f(s)\|_{q} \leq \varepsilon \quad \text { and } \quad s^{\alpha / 2}\|f(s)\|_{q} \leq \varepsilon,
$$


for $0 \leq s<h$, then an argument analogous to the one used in (1.28)(1.30) shows that (for $0 \leq s<h$ )

$$
\|B(f, g)(t)\|_{3} \leq \varepsilon
$$

which is nothing more than the $(\varepsilon, h)$ definition of $(1.36)$. The proof of (1.34) is essentially the same and does not present any difficulties. The Lemma 1.4 follows.

Let us now recall without proof, a classical result.

Lemma 1.5. Let $X$ be an abstract Banach space and $B: X \times X \longrightarrow X$ a bilinear operator, $\|\cdot\|$ being the $X$-norm, such that for any $x_{1} \in X$ and $x_{2} \in X$, we have

$$
\left\|B\left(x_{1}, x_{2}\right)\right\| \leq \eta\left\|x_{1}\right\|\left\|x_{2}\right\|
$$

then for any $y \in X$ such that

$$
4 \eta\|y\|<1,
$$

the equation

$$
x=y+B(x, x)
$$

has a solution $x$ in $X$. Moreover, this solution $x$ is the only one such that

$$
\|x\| \leq \frac{1-\sqrt{1-4 \eta\|y\|}}{2 \eta} .
$$

The proof of Theorem 1.1 now easily follows if we take into account all the previous lemmata.

\section{A remarkable property.}

In order to appreciate the above-mentioned weak formulation of Kato's theorem, in this Section, we shall devote ourselves to illustrating that the condition $\left\|v_{0}\right\|_{\dot{B}_{q}^{-\alpha, \infty}}<\delta$ is satisfied in the particular case of a sufficiently oscillating function $v_{0}$. 
A typical situation will be given by the following example. Let $v_{0}$ be an arbitrary (not identically vanishing) function belonging to $L^{3}\left(\mathbb{R}^{3}\right)$. If we multiply $v_{0}$ by an exponential, say the function $w_{k}=$ $\exp (i x \cdot k)$, we obtain, for any $k \in \mathbb{R}^{3}$, a function $w_{k} v_{0}$ such that (Lemma 2.1)

$$
\lim _{|k| \rightarrow \infty}\left\|w_{k} v_{0}\right\|_{\dot{B}_{q}^{-\alpha, \infty}}=0
$$

in spite of the fact that

$$
\lim _{|k| \rightarrow \infty}\left\|w_{k} v_{0}\right\|_{3}=\left\|v_{0}\right\|_{3}
$$

In other words, the smallness condition $\left\|w_{k} v_{0}\right\|_{\dot{B}_{q}^{-\alpha, \infty}}<\delta$, is verified as long as we choose a sufficiently high frequency $k$. At this point, it is tempting to consider $w_{k} v_{0}$ as the new initial data of the problem and affirm that Kato's solution exists globally in time, provided we consider a sufficiently oscillating data; but one can argue that $w_{k} v_{0}$ is no longer a divergence-free function.

Nevertheless, this is true asymptotically, for $|k| \rightarrow \infty$, which is exactly the situation we are dealing with. More precisely, it turns out that (Lemma 2.2)

$$
\lim _{|k| \rightarrow \infty}\left\|\nabla \cdot\left(w_{k} v_{0}\right)-w_{k} \nabla \cdot v_{0}\right\|_{3}=0
$$

Lemma 2.1. Let $v$ be an arbitrary function in $L^{3}\left(\mathbb{R}^{3}\right)$ and let $w_{k}(x)$, $k \in \mathbb{N}$ be a sequence of functions such that $\left\|w_{k}\right\|_{\infty} \leq C$ and $w_{k} \rightarrow 0$ (as $k \rightarrow \infty)$ in the distributional sense. Then, the products $w_{k} v$ tend to 0 in the strong topology of $\dot{B}_{q}^{-\alpha, \infty}\left(\mathbb{R}^{3}\right)(\alpha=1-3 / q>0)$.

In order to prove the lemma we will make use of a density argument. To this end let us introduce the following decomposition of the function $v$,

$$
v=h+g
$$

where $h \in L^{3}\left(\mathbb{R}^{3}\right)$ and

$$
\|h\|_{3} \leq \varepsilon
$$


and $g \in C_{0}^{\infty}\left(\mathbb{R}^{3}\right)$. The next step is to recall the continuous imbedding (Lemma 1.2).

$$
L^{3}\left(\mathbb{R}^{3}\right) \hookrightarrow \dot{B}_{q}^{-\alpha, \infty}\left(\mathbb{R}^{3}\right)
$$

to infer the following inequality $(k \geq 0)$

$$
\left\|w_{k} h\right\|_{\dot{B}_{q}^{-\alpha, \infty}} \leq c\left\|w_{k} h\right\|_{3} \leq c \varepsilon .
$$

On the other hand, Young's inequality gives $(j \in \mathbb{Z})$

$$
\left\|S_{j}\left(w_{k} g\right)\right\|_{q} \leq\left\|2^{3 j} \varphi\left(2^{j} \cdot\right)\right\|_{r}\left\|w_{k} g\right\|_{p},
$$

where

$$
\frac{1}{q}=\frac{1}{r}+\frac{1}{p}-1
$$

This implies

$$
\begin{aligned}
2^{-\alpha j}\left\|S_{j}\left(w_{k} g\right)\right\|_{q} & \leq C 2^{-j(1-3 / q)} 2^{-j(1-3 / r)}\|g\|_{p} \\
& =C 2^{-j(1-3 / p)}\|g\|_{p},
\end{aligned}
$$

so that, for any $k \geq 0$, any $j \geq j_{1}>0$ and any $j \leq j_{0}<0$, we have

$$
2^{-\alpha j}\left\|S_{j}\left(w_{k} g\right)\right\|_{q} \leq C \varepsilon
$$

(in fact, if $j \geq j_{1}$ we let $p=q>3$ and if $j \leq j_{0}$ we let $1 \leq p<3$ ).

We are now left with the terms $S_{j}\left(w_{k} g\right)$ for $j_{0}<j<j_{1}$. Making use of the hypothesis $m_{k} \rightarrow 0$ together with the Lebesgue dominated convergence theorem, we finally find, for any $k \geq k_{0}$ and $j_{0}<j<j_{1}$,

$$
2^{-\alpha j}\left\|S_{j}\left(w_{k} g\right)\right\|_{q} \leq C \varepsilon,
$$

which concludes the proof of the Lemma.

Lemma 2.2. Let $m(\xi) \in C^{\infty}\left(\mathbb{R}^{3} \backslash\{0\}\right)$ be a homogeneous function of degree 0 and let $M$ be the convolution operator associated to the multiplier $m(\xi)$. If now we consider $\left|\xi_{0}\right|=1, v \in L^{p}\left(\mathbb{R}^{3}\right)$ and $1<p<$ $\infty$, then

$$
\begin{aligned}
\lim _{\lambda \rightarrow \infty} \sup _{\left|\xi_{0}\right|=1} \| & M\left(\exp \left(i \lambda \xi_{0} \cdot x\right) v(x)\right) \\
& -\exp \left(i \lambda \xi_{0} \cdot x\right) m\left(\xi_{0}\right) v(x) \|_{p}=0 .
\end{aligned}
$$


In the case we are interested in, this Lemma will be used for $p=3$ and with $M$ replaced by the projection operator $\mathbb{P}$ onto the divergence free vector fields and $m(\xi)$ replaced by a $3 \times 3$ matrix whose entries are homogeneous symbols of degree 0 .

In order to prove the Lemma in its general form, we remark that the symbol of the operator $\exp \left(-i \lambda \xi_{0} \cdot x\right) M\left(\exp \left(i \lambda \xi_{0} \cdot x\right) v\right)-m\left(\xi_{0}\right) v(x)$ is given by $m\left(\xi+\lambda \xi_{0}\right)-m\left(\lambda \xi_{0}\right)$, this by virtue of the homogeneity of $m$.

Equation (2.13) will now be proved by means of a density argument. In fact, it is sufficient to limit ourselves to functions $v \in \mathcal{V} \subset$ $L^{p}\left(\mathbb{R}^{3}\right)$, where $\mathcal{V}$ is the dense subspace of $L^{p}\left(\mathbb{R}^{3}\right)$ defined by $v \in \mathcal{S}\left(\mathbb{R}^{3}\right)$ and the Fourier transform $\hat{v}$ of $v$ has compact support.

Now, we put

$$
v_{\lambda}=\exp \left(-i \lambda \xi_{0} \cdot x\right) M\left(\exp \left(i \lambda \xi_{0} \cdot x\right) v\right)-m\left(\lambda \xi_{0}\right) v
$$

then the Fourier transform of $v_{\lambda}$ is given by

$$
\hat{v_{\lambda}}(\xi)=\left(m\left(\xi+\lambda \xi_{0}\right)-m\left(\lambda \xi_{0}\right)\right) \hat{v}(\xi) \text {. }
$$

Finally, $\hat{v}$ has compact support, say in $|\xi| \leq R$, and then

$$
m\left(\xi+\lambda \xi_{0}\right)-m\left(\lambda \xi_{0}\right)=r_{\lambda}(\xi)
$$

where, on $|\xi| \leq R, r_{\lambda}(\xi) \rightarrow 0$, together with all its derivates, in the $L^{\infty}$ norm. We thus have $v_{\lambda} \rightarrow 0$ in $\mathcal{S}\left(\mathbb{R}^{3}\right)$ when $\lambda \rightarrow \infty$. A fortiori, $\left\|v_{\lambda}\right\|_{p} \rightarrow 0$ when $\lambda \rightarrow \infty$, and the Lemma is proved.

\section{Self-similar solutions.}

As we pointed out in the Introduction, a remarkable property of the Navier-Stokes equations

$$
\left\{\begin{array}{l}
\frac{\partial v}{\partial t}-\Delta v=-(v \cdot \nabla) v-\nabla p \\
\nabla \cdot v=0
\end{array}\right.
$$

is that they are invariant under the scaling $(v, p)$ implies $\left(v_{\lambda}, p_{\lambda}\right)$ for all $\lambda>0$, where, respectively,

$$
v_{\lambda}(t, x)=\lambda v\left(\lambda^{2} t, \lambda x\right)
$$


and

$$
p_{\lambda}(t, x)=\lambda^{2} p\left(\lambda^{2} t, \lambda x\right)
$$

In other words, suppose that $(v, p)$ is a solution of the system (3.1), then the same holds true for $\left(v_{\lambda}, p_{\lambda}\right)$ for any $\lambda>0$.

An interesting question arises naturally. Are there solutions $v(t, x)$ of the Navier-Stokes equations which satisfy the scaling invariance

$$
v(t, x)=v_{\lambda}(t, x), \quad \text { for all } \lambda>0 ?
$$

Whenever they exist, these particular solutions are called self-similar solutions to the Navier-Stokes equations and are, by definition, global in time.

Self-similar solutions are important because they describe the large time behavior of general global solutions to (3.1). A heuristic argument for this property is the following. Suppose that $v(t, x)$ is a global solution to (3.1), then $v_{\lambda}(t, x)=\lambda v\left(\lambda^{2} t, \lambda x\right)$ is, for any $\lambda>0$, a solution to (3.1). If, moreover, we suppose that $\lim _{\lambda \rightarrow \infty} v_{\lambda}(t, x)=u(t, x)$ exists (in a certain sense), then $u(t, x)$ is again a solution of the system (3.1) and, by taking $t=1$ and $\lambda=\sqrt{t}$, we have $\lim _{t \rightarrow \infty} \sqrt{t} v(t, \sqrt{t} x)=u(1, x)$.

Here is another remarkable property of self-similar solutions. Suppose that $v(t, x)$ is a self-similar solution, then the value taken by $v(t, x)$ at $t=0$, say the function $v(0, x)$, is necessary homogeneous of degree -1 and has divergence zero (in the sense of distributions). The explicit aim of this Section is to check whether or not the converse is true. More precisely, given a homogeneous function $v_{0}$ of degree -1 , whose divergence is zero (in the sense of distributions), does the Cauchy problem

$$
\left\{\begin{array}{l}
\frac{\partial v}{\partial t}-\Delta v=-(v \cdot \nabla) v-\nabla p \\
\nabla \cdot v=0 \\
v(0)=v_{0}
\end{array}\right.
$$

associated to the system (3.1) admit a self-similar solution $v(t, x)$ ?

As we will discover in due course, the answer to this problem is positive, provided we choose a suitable functional setting for the problem.

It is worthwhile to recall that the above question is in general far from being trivial in $\mathbb{R}^{3}$ because, as is well-known, a uniqueness result for the Navier-Stokes equation is still lacking in dimension $n \geq 3$. 
To clarify, suppose to the contrary that we dispose of such a uniqueness theorem for the solutions of the system (3.5) and suppose that, moreover, for $v_{0}$ homogeneous of degree $-1, \nabla \cdot v_{0}=0$, we were able to prove that the solution of the initial value problem (3.5) actually exists. Under these hypothesis, such a solution would be automatically self-similar.

On the other hand, the fact that $v_{0}$ should be homogeneous of degree -1 excludes practically all the classical spaces (e.g. Lebesgue and Sobolev) for which at least an existence theorem for the NavierStokes equations is available. As a matter of fact, if we forget for a while the divergence condition, the simplest example of a homogeneous function of degree -1 is given by $|x|^{-1}, x=\left(x_{1}, x_{2}, x_{3}\right)$, which does not belong to any Lebesgue nor Sobolev spaces.

The problem of finding self-similar solutions for the Navier-Stokes equations was tackled as early as 1933 by J. Leray in his pioneering Ph. D. dissertation [14]. More precisely, Leray was interested in the apparition of possible singularities for solutions $v(t, x)$ of the particular form

$$
v(t, x)=\lambda(t) V(\lambda(t) x)
$$

$\lambda(t)$ being a positive scalar function and $V(x)$ being a divergence-free vector field.

It is clear that if $v(t, x)$ is a self-similar solution of the NavierStokes equations, in other words, if $v(t, x)=\lambda v\left(\lambda^{2} t, \lambda x\right)$, for all $\lambda>0$, then by taking $\lambda=\sqrt{t}$ we find, in Leray's notation,

$$
v(t, x)=\frac{1}{\sqrt{t}} V\left(\frac{x}{\sqrt{t}}\right)
$$

where $V(x)=v(1, x)$ is an arbitrary divergence-free vector field.

After Leray's work, this particular representation, equation (3.7), was utilized by several authors with the aim of finding self-similar solutions by a direct approach, say via the elliptic equation (in the unknown functions $V$ and $Q$ )

$$
\left\{\begin{array}{l}
-\frac{1}{2} V-\frac{1}{2}(x \cdot \nabla) V-\Delta V=-(V \cdot \nabla) V-\nabla Q \\
\nabla \cdot V=0
\end{array}\right.
$$

obtained by substituting (3.7) into (3.1). 
Unfortunately, this strategy turned out to be unsuccessful (see, for a detailed discussion [15]) and the system (3.8) too difficult to solve.

The problem of finding self-similar solutions was still completely open when, in 1989, Y. Giga and T. Miyakawa [16] showed that in a suitable Morrey-Campanato space, self-similar solutions to the NavierStokes equations written in terms of the vorticity $\omega(t, x)=\operatorname{curl} v(t, x)$ exist as long as the initial data $\omega_{0}(x)$ are homogeneous of degree -2 and small enough.

In this Section, we tackle and solve the problem in a somewhat different way. More specifically, we will not deal with the elliptic equation (3.8), nor with the Navier-Stokes equations with vorticity as an unknown function but, in the previous notations, we look for self-similar solutions $v(t, x)$ of the mild equation

$$
v(t)=S(t) v_{0}+B(v, v)(t)
$$

where

$$
B(v, u)(t)=-\int_{0}^{t} \mathbb{P} S(t-s) \nabla \cdot(v \otimes u)(s) d s .
$$

The idea pursued here is to establish first a general existence and uniqueness theorem for mild solutions $v(t, x) \in C([0, \infty) ; X)$ of $(3.9)$, $X$ being an abstract Banach space containing homogeneous functions of degree -1 , and then obtain the existence of self-similar solutions as a corollary.

In Section 1, we showed that the Besov spaces $\dot{B}_{q}^{-\alpha, \infty}\left(\mathbb{R}^{3}\right)(3<$ $q<\infty, \alpha=1-3 / q$ ) have the remarkable property of allowing among their elements homogeneous functions of degree -1 . Moreover, these spaces arise in a natural generalization of Kato's theorem (Theorem $1.1)$.

The starting point of this Part 3 is to restate this theorem in the full Besov setting, i.e. to remove the condition $v_{0} \in L^{3}\left(\mathbb{R}^{3}\right)$. This will be a crucial step in the proof of the existence of self-similar solutions, because, for instance, $|x|^{-1} \notin L^{3}\left(\mathbb{R}^{3}\right)$. Some technical difficulties will appear when passing from $L^{3}\left(\mathbb{R}^{3}\right)$ to $\dot{B}_{q}^{-\alpha, \infty}\left(\mathbb{R}^{3}\right)$, because the latter Banach space is not separable. This implies, among other things, that the property (1.21) (which plays an important role in the uniqueness part of Theorem 1.1) is no longer verified when $v(t)$ is replaced by $S(t) v_{0}, v_{0} \in \dot{B}_{q}^{-\alpha, \infty}\left(\mathbb{R}^{3}\right)$. An easy way to see this is to consider, for example,

$$
v_{0}(x)=\left(0, \frac{-x_{3}}{|x|^{2}}, \frac{x_{2}}{|x|^{2}}\right)
$$


and remark that

$$
\lim _{t \rightarrow 0} t^{\alpha / 2}\left\|S(t) v_{0}\right\|_{q}=\left\|S(1) v_{0}\right\|_{q} \neq 0
$$

Another important limitation imposed when dealing with a non-separable Banach space $X$ is that the heat semigroup is no longer a $\mathcal{C}_{0^{-}}$ semigroup. This means that $S(t) v_{0}$ is no longer a strongly continuous function from $[0, \infty)$ into $X$. A way to circumvent this difficulty is to replace the space $C([0, \infty) ; X)$ by the space $C_{*}([0, \infty) ; X)$ whose elements $v(t, x)$ are bounded flows in $X$, viz. $v(t, x) \in L^{\infty}([0, \infty) ; X)$ and are continuous in the weak sense of distributions.

With this modification in mind, and recalling that the standard fixed point algorithm (Lemma 1.5) gives the uniqueness of the solution in a small neighborhood of the origin, we will obtain the following result.

Theorem 3.1. Let $q$ be fixed in $3<q \leq 6$ and $\alpha=\alpha(q)=1-$ $3 / q$, then there exists an absolute constant $\delta>0$ such that if $v_{0}$ belongs to $\dot{B}_{q}^{-\alpha, \infty}\left(\mathbb{R}^{3}\right),\left\|v_{0}\right\|_{\dot{B}_{q}^{-\alpha, \infty}}<\delta$ and $\nabla \cdot v_{0}=0$ (in the sense of distributions), then there exists a global mild solution of the NavierStokes equations such that

$$
\begin{gathered}
v(t, x) \in C_{*}\left((0, \infty) ; \dot{B}_{q}^{-\alpha, \infty}\left(\mathbb{R}^{3}\right)\right), \\
t^{\alpha / 2} v(t, x) \in C_{*}\left((0, \infty) ; L^{q}\left(\mathbb{R}^{3}\right)\right)
\end{gathered}
$$

and, if $3<q \leq 4$,

$$
v(t, x)-S(t) v_{0} \in C_{*}\left((0, \infty) ; \dot{H}^{1 / 2}\left(\mathbb{R}^{3}\right)\right)
$$

and, if $4<q \leq 6$,

$$
v(t, x)-S(t) v_{0} \in C_{*}\left((0, \infty) ; L^{3}\left(\mathbb{R}^{3}\right)\right) .
$$

Moreover, there exists only one solution $v(t, x)$ verifying (3.13)-(3.14) and such that

$$
\sup _{t \geq 0}\|v(t)\|_{\dot{B}_{q}^{-\alpha, \infty}}+\sup _{t \geq 0} t^{\alpha / 2}\|v(t)\|_{q} \leq R
$$

where $R=R\left(\left\|v_{0}\right\|_{\dot{B}_{q}^{-\alpha, \infty}}\right)$ is a given constant. 
The proof of Theorem 3.1 is essentially the same of that presented for Theorem 1.1. Here, equation (3.16) follows from an argument similar to the one given in (1.30).

The only point which merits clarification is the regularity property (3.15). To see this, let us recall the simplified bilinear scalar operator

$$
B(f, g)(t)=-\int_{0}^{t}(t-s)^{-2} \Theta\left(\frac{\cdot}{\sqrt{t-s}}\right) *(f g)(s) d s .
$$

Next, in order to evaluate the $\dot{H}^{1 / 2}\left(\mathbb{R}^{3}\right)$-norm of $B(f, g)(t)$, let us consider the operator $\Lambda^{1 / 2}$ (whose symbol is $|\xi|^{1 / 2}$ ), where $\Lambda=(-\Delta)^{1 / 2}$ is the usual Calderón operator.

We find

$$
\Lambda^{1 / 2} B(f, g)(t)=c \int_{0}^{t}(t-s)^{-9 / 4} \Theta_{1}\left(\frac{\cdot}{\sqrt{t-s}}\right) *(f g)(s) d s
$$

where $\Theta_{1}=\Lambda^{\frac{1}{2}} \Theta \in L^{1}\left(\mathbb{R}^{3}\right) \cap L^{\infty}\left(\mathbb{R}^{3}\right)$. Finally, if $3<q \leq 4$, we obtain

$$
\begin{aligned}
\left\|\Lambda^{1 / 2} B(f, g)(t)\right\|_{2} \leq & c\left(\int_{0}^{t}(t-s)^{-9 / 4+3 /(2 m)} s^{-\alpha} d s\right)\left\|\Theta_{1}\right\|_{m} \\
& \cdot\left(\sup _{t \geq 0} t^{\alpha / 2}\|f(t)\|_{q}\right)\left(\sup _{t \geq 0} t^{\alpha / 2}\|g(t)\|_{q}\right),
\end{aligned}
$$

where

$$
\frac{1}{2}=\frac{1}{m}+\frac{2}{q}-1
$$

and the estimate (3.15) follows.

As announced before, it is now elementary to obtain, as a particular case of Theorem 3.1, the following existence and uniqueness result of self-similar solutions for the Navier-Stokes equations. Here, the crucial point is that condition (3.17) is invariant under the transformation $v(t, x)$ implies $v_{\lambda}(t, x)$ for all $\lambda>0$.

Theorem 3.2. Let $q$ be fixed in $3<q \leq 6$ and $\alpha=\alpha(q)=1-3 / q$, then there exists an absolute constant $\delta>0$ such that if $v_{0}$ belongs to $\dot{B}_{q}^{-\alpha, \infty}\left(\mathbb{R}^{3}\right),\left\|v_{0}\right\|_{\dot{B}_{q}^{-\alpha, \infty}}<\delta, \nabla \cdot v_{0}=0$ (in the sense of distributions) and $v_{0}(x)=\lambda v_{0}(\lambda x)$ for all $\lambda>0$, then there exists a global mild solution of the Navier-Stokes equations which is written in the form

$$
v(t, x)=\frac{1}{\sqrt{t}} V\left(\frac{x}{\sqrt{t}}\right),
$$


where $V \in \dot{B}_{q}^{-\alpha, \infty}\left(\mathbb{R}^{3}\right) \cap L^{q}\left(\mathbb{R}^{3}\right)$ is such that

$$
V(x)=S(1) v_{0}+W(x)
$$

with $W \in \dot{H}^{1 / 2}\left(\mathbb{R}^{3}\right)$, if $3<q \leq 4$, and $W \in L^{3}\left(\mathbb{R}^{3}\right)$, if $4<q \leq 6$. The initial value $v_{0}$ is taken by $v(t, x)$ at least in the weak sense of distributions. Finally, there is only one solution $v(t, x)$ such that $V \in$ $\dot{B}_{q}^{-\alpha, \infty}\left(\mathbb{R}^{3}\right) \cap L^{q}\left(\mathbb{R}^{3}\right)$ and

$$
\|V\|_{\dot{B}_{q}^{-\alpha, \infty}}+\|V\|_{q} \leq R
$$

where $R=R\left(\left\|v_{0}\right\|_{\dot{B}_{q}^{-\alpha, \infty}}\right)$ is a given constant.

\section{An equivalence theorem for homogeneous functions on Besov spaces.}

Starting from the remark that a homogeneous function of degree -1 is known in all $\mathbb{R}^{3}$ by its restriction on the unit sphere $S^{2}$, in this Section we present an equivalence theorem for homogeneous functions of degree -1 which belong to the Besov space $\dot{B}_{q}^{-\alpha, \infty}\left(\mathbb{R}^{3}\right)$, with $1 \leq q \leq \infty$ and $\alpha=1-3 / q$. In Section 1 (Lemma 1.2), we showed that it is sufficient for a homogeneous function $f$ of degree -1 to have an $L^{\infty}\left(S^{2}\right)$ restriction to the unit sphere $S^{2}$, to ensure that $f \in \dot{B}_{q}^{-\alpha, \infty}\left(\mathbb{R}^{3}\right), q>$ $3, \alpha=1-3 / q$.

This remark is enough to guarantee that both Theorems 3.1 and 3.2 do not admit only the trivial data $v_{0}=0$ as initial condition. In fact if we consider

$$
v_{0}=\left(0, \frac{-x_{3}}{|x|^{2}}, \frac{x_{2}}{|x|^{2}}\right)
$$

then $v_{0}$ is divergence free and belongs to $\dot{B}_{q}^{-\alpha, \infty}\left(\mathbb{R}^{3}\right)$ for $q>3$ and $\alpha=1-3 / q$.

In this Section we want to prove the following more general and accurate result.

Theorem 4.1. Let $1 \leq q \leq \infty$ and $\alpha=1-3 / q$ be fixed. Then, for any homogeneous distribution $f$ of degree -1 the following three conditions are equivalent

i) $f$ belongs to the homogeneous Besov space $\dot{B}_{q}^{-\alpha, \infty}\left(\mathbb{R}^{3}\right)$, 
ii) the restriction of $f$ to a certain neighborhood $\Omega$ of the unit sphere $S^{2}$ belongs to the non-homogeneous space $B_{q}^{-\alpha, q}(\Omega)$,

iii) the restriction of $f$ to the unit sphere $S^{2}$ belongs to the nonhomogeneous Besov space $B_{q}^{-\alpha, q}\left(S^{2}\right)$.

Before delving into the details of the proof of this theorem, let us recall some simple properties of the homogeneous Besov spaces $B_{q}^{s, p}\left(\mathbb{R}^{3}\right)$, for $(s, p, q) \in \mathbb{R} \times[1, \infty] \times[1, \infty]$.

First of all $B_{q}^{s, p}\left(\mathbb{R}^{3}\right)$ is a module in the ring $C_{0}^{\infty}\left(\mathbb{R}^{3}\right)$. This means that the elements $\lambda$ of $C_{0}^{\infty}\left(\mathbb{R}^{3}\right)$ define endomorphisms $\lambda f\left(\lambda \in C_{0}^{\infty}\left(\mathbb{R}^{3}\right)\right.$, $\left.f \in B_{q}^{s, p}\left(\mathbb{R}^{3}\right)\right)$ of $B_{q}^{s, p}\left(\mathbb{R}^{3}\right)$, as in the case of a vector space. The second remark is that it is now possible to define a local space $B_{q}^{s, p}$. The localization (in a neighborhood of $x_{0}$ ) is obtained by multiplying by a cut-off function $\chi \in C_{0}^{\infty}\left(\mathbb{R}^{3}\right)$ equal to one in a neighborhood of $x_{0}$.

The second group of observations concerns the possibility of extending a function $f(x) \in B_{q}^{s, p}\left(\mathbb{R}^{2}\right)$ into a function $\tilde{f}(x, y)$ that we can suppose to be either independent of $y$ and equal to $f(x)$ if $y=0$, or equal to $f(x) \varphi(y)$, where $\varphi$ is the function appearing in a LittlewoodPaley decomposition and is such that $\varphi(0) \neq 0$. These two points of view are equivalent in a neighborhood of the $\mathbb{R}^{2} \times\{0\}$, because we can multiply by $1 / \varphi(y)$ in a neighborhood of $\mathbb{R}^{2} \times\{0\}$.

Let us start with a proof of Theorem 4.1 in a model global case as given by the following Lemma.

Lemma 4.1. Let $g \in S\left(\mathbb{R}^{3}\right)$ be a function whose Fourier transform $\hat{g}$ has compact support. To fix ideas, let $g(0) \neq 0$. Then, for any function $f\left(\right.$ defined on $\left.\mathbb{R}^{2}\right)$ and any set of index $(s, p, q) \in \mathbb{R} \times[1, \infty] \times[1, \infty]$, the norm of $f(x)$ in the nonhomogeneous Besov space $B_{q}^{s, p}\left(\mathbb{R}^{2}\right)$ is equivalent to that of $f(x) g(y)$ in $B_{q}^{s, p}\left(\mathbb{R}^{3}\right)$

Here is a simple proof of this result. First of all, let us consider a Littlewood-Paley decomposition in $\mathbb{R}$ associated to a function $\varphi$ such that the support of $\hat{g}$ is included in the set of points $y \in \mathbb{R}$ such that $\hat{\varphi}(y)=1$. Then, we use a Littlewood-Paley decomposition in $\mathbb{R}^{3}$ associated to the product structure. In other words,

$$
\begin{aligned}
I & =S_{0} \otimes S_{0}+\sum_{0}^{\infty}\left(S_{j+1} \otimes S_{j+1}-S_{j} \otimes S_{j}\right) \\
& =S_{0} \otimes S_{0}+\sum_{0}^{\infty}\left(S_{j} \otimes \Delta_{j}+\Delta_{j} \otimes S_{j}+\Delta_{j} \otimes \Delta_{j}\right) .
\end{aligned}
$$


But $S_{j}(g)=g$ for $j \geq 0$ and $\Delta_{j} g=0$ for $j \geq 0$. This implies that $\left(\Delta_{j} \otimes\right.$ $\left.S_{j}\right)(f g)=\left(\Delta_{j} f\right)\left(S_{j} g\right)=\left(\Delta_{j} f\right) g$ and $\left(S_{j} \otimes \Delta_{j}\right)(f g)=\left(S_{j} f\right)\left(\Delta_{j} g\right)=0$ for $j \geq 0$. Finally,

$$
\begin{aligned}
\left(\sum_{0}^{\infty} 2^{p j s}\left\|\Delta_{j}(f g)\right\|_{q}^{p}\right)^{1 / p} & =\left(\sum_{0}^{\infty} 2^{p j s}\left\|\Delta_{j} f\right\|_{q}^{p}\|g\|_{q}^{p}\right)^{1 / p} \\
& =\|g\|_{q}\left(\sum_{0}^{\infty} 2^{p j s}\left\|\Delta_{j} f\right\|_{q}^{p}\right)^{1 / p}
\end{aligned}
$$

which concludes the proof of the Lemma.

Before proving Theorem 4.1 in all its generality, we recall here an other interesting result

Lemma 4.2. Let $S^{2} \subset \mathbb{R}^{3}$ be the unit sphere. For any tempered distribution $f \in D^{\prime}\left(S^{2}\right)$, let $\tilde{f}$ designate the distribution defined in the open set $\Omega=\left\{x \in \mathbb{R}^{3}: 1 / 2<|x|<3 / 2\right\}$ by

$$
\tilde{f}(x)=f\left(\frac{x}{|x|}\right) \chi(|x|),
$$

where $\chi \in C_{0}^{\infty}[1 / 2,3 / 2]$ and $\chi=1$ in a neighborhood of 1 . With these notations, for any $(s, p, q) \in \mathbb{R} \times[1, \infty] \times[1, \infty]$ we have

$$
f \in B_{q}^{s, p}\left(S^{2}\right) \quad \text { if and only if } \quad \tilde{f} \in B_{q}^{s, p}(\Omega) .
$$

The proof of this result is trivial, because $S^{2}$ is compact, so that one can first argue locally in a neighborhood of the point $x_{0} \in S^{2}$ and conclude in a standard way. The details are left to the reader.

We are now in a position to prove Theorem 4.1. The equivalence ii) if and only if iii) is a consequence of Lemma 4.2. In order to prove that i) if and onlt if ii) we use the well-known wavelet characterization of the homogeneous Besov spaces [13]. More precisely, let $\psi(x)=\psi_{\varepsilon}(x), x \in$ $\mathbb{R}^{3}$ designate the mother wavelets $(\varepsilon=1,2, \ldots, 7)$ and let us consider a Daubechies compactly supported orthonormal base of $\mathbb{R}^{3}$. Let us put

$$
c(j, k)=\int_{\mathbb{R}^{3}} 2^{3 j} \bar{\psi}\left(2^{j} x-k\right) f(x) d x
$$

the wavelet coefficients of a function $f$, the normalization being intended in the $L^{1}\left(\mathbb{R}^{3}\right)$ sense. Then, the following characterization holds [13] 
Lemma 4.3. $f \in \dot{B}_{q}^{s, p}\left(\mathbb{R}^{3}\right)$ if and only if

$$
\sum_{j \in \mathbb{Z}}\left(\sum_{k \in \mathbb{Z}^{3}}|c(j, k)|^{q} 2^{s j q} 2^{-3 j}\right)^{p / q}<\infty .
$$

We will use this lemma with $s=-\alpha=3 / q-1, p=\infty$ and $f$ homogeneous of degree -1 . Now, taking into account this last property, we can write

$$
c(j, k)=2^{j} c(0, k)=: 2^{j} c(k)
$$

so that condition (4.7) becomes

$$
f \in \dot{B}_{q}^{-\alpha, \infty}\left(\mathbb{R}^{3}\right) \quad \text { if and only if } \quad c(k) \in l^{q}\left(\mathbb{Z}^{3}\right) .
$$

Finally, we consider the neighborhood $\Omega=\left\{x \in \mathbb{R}^{3}: 1 / 2<|x|<3 / 2\right\}$ of the unit sphere $S^{2}$ and evaluate the $B_{q}^{-\alpha, q}(\Omega)$ norm of an arbitrary function $f$ by using the wavelets whose support is included in $\Omega$. This means $1 / 2<\left|k 2^{-j}\right|<3 / 2$ and $j \geq j_{0}$. The last step in the proof of Theorem 4.1 is given by the following well-known result [17]

Lemma 4.4 Let $\Omega$ be an open bounded set in $\mathbb{R}^{3}$. Let us suppose that $f$ belongs to $B_{q}^{s, p}(\Omega)$, in other words, let us suppose that $f$ is the restriction to $\Omega$ of a function in $B_{q}^{s, p}\left(\mathbb{R}^{3}\right)$. Then we have

$$
\sum_{j}\left(\sum_{k}|c(j, k)|^{q} 2^{s j q} 2^{-3 j}\right)^{p / q}<\infty
$$

where the sum over $j$ and $k$ is restricted to the values for which the support $S(j, k)$ of $\psi_{j, k}=2^{3 j} \psi\left(2^{j} x-k\right)$ is included in $\Omega$. If, conversely, this condition is verified, then for any $\Omega_{\varepsilon} \subset \Omega$, with $d\left(\Omega_{\varepsilon}, \Omega^{c}\right) \geq \varepsilon, f$ belongs to $B_{q}^{s, p}\left(\Omega_{\varepsilon}\right)$.

Let us conclude the proof of Theorem 4.1. If $f$ is homogeneous of degree -1 , then $f \in B_{q}^{-\alpha, q}(\Omega)$ means

$$
\sum_{j \geq j_{0}} \sum_{1 / 2 \leq\left|k 2^{-j}\right| \leq 3 / 2}|c(k)|^{q}=\sum_{k \in \mathbb{Z}^{3}}|c(k)|^{q}<\infty,
$$

that is $c(k) \in l^{q}\left(\mathbb{Z}^{3}\right)$, which, in turn, is equivalent to $f \in \dot{B}_{q}^{-\alpha, \infty}\left(\mathbb{R}^{3}\right)$. Here the crucial point is that the dyadic coronas $1 / 2 \leq\left|k 2^{-j}\right| \leq 3 / 2$ cover exactly all of $\mathbb{Z}^{3} \backslash\{0\}$. The proof of Theorem 4.1 is now completed. 
Before ending, let us remark, as announced, (see also [1]) that if a function $f$ homogeneous of degree -1 is such that its restriction to the unit sphere $S^{2}$ is bounded, then

$$
\sum_{0}^{\infty} 2^{(3-q) j}\left\|\Delta_{j} f\right\|_{L^{q}\left(S^{2}\right)}^{q} \leq c \sum_{0}^{\infty} 2^{(3-q) j}=c
$$

as long as $q>3$, which implies that $f \in \dot{B}_{q}^{-\alpha, \infty}\left(\mathbb{R}^{3}\right)$. More generally, according to a celebrated result of J. E. Littlewood and R. Paley [18], the sufficient condition

$$
\left.f\right|_{S^{2}} \in L^{q}\left(S^{2}\right)
$$

would give (for $q>3$ ) the same result, for

$$
\sum_{0}^{\infty} 2^{(3-q) j}\left\|\Delta_{j} f\right\|_{L^{q}\left(S^{2}\right)}^{q} \leq c \sum_{0}^{\infty}\left\|\Delta_{j} f\right\|_{L^{q}\left(S^{2}\right)}^{q} \leq c .
$$

Acknowledgements. This article is based on the author's Ph.D thesis. The author expresses his gratitude to his advisor Y. Meyer for his constant support and generous help. The kind hospitality received at the Department of Mathematics of Washington University in St. Louis, where this work was written under a post-doctoral fellowship, is also gratefully acknowledged.

\section{References.}

[1] Cannone, M., Ondelettes, paraproduits et Navier-Stokes. Diderot Editeur, 1995.

[2] Kato, T., Strong $L^{p}$ solutions of the Navier-Stokes equations in $\mathbb{R}^{m}$ with applications to weak solutions. Math. Z. 187 (1984), 471-480.

[3] Giga, Y., Solutions for semilinear parabolic equations in $L^{p}$ and regularity of weak solutions of the Navier-Stokes system. J. Differential Equat. 61 (1986), 186-212.

[4] Fujita, H., Kato. T., On the Navier-Stokes initial value problem I. Arch. Rational Mech. Anal. 16 (1964), 269-315.

[5] Chemin, J. Y., Remarques sur l'existence globale pour le système de Navier-Stokes incompressible. SIAM J. Math. Anal. 23 (1992), 20-28. 
[6] Federbush, P., Navier and Stokes meet the wavelet. Comm. Math. Phys. 155 (1993), 219-248.

[7] Taylor, M. E., Analysis on Morrey spaces and applications to the NavierStokes and other evolution equations. Comm. Partial Diff. Equations. 17 (1992), 1407-1456.

[8] Kato, T., Strong solutions of the Navier-Stokes equations in Morrey spaces. Bol. Soc. Brasil. Math. (N.S.) 22 (1992), 127-155.

[9] Kozono, H., Yamazaki, M., Semilinear heat equations and the NavierStokes equation with distributions in new function spaces as initial data. Comm. Partial Diff. Equations. 19 (1994), 959-1014.

[10] Cannone, M., Meyer, Y., Littlewood-Paley decomposition and NavierStokes equations. Meth. and Appl. of Anal. 2 (1995), 307-319.

[11] Frazier, M., Jawerth, B., Weiss, G., Littlewood-Paley theory and the study of function spaces. Monograph in the CBM-AMS 79, 1991.

[12] Frazier, M., Jawerth, B., A discrete transform and decompositions of distribution spaces. J. Funct. Anal. 93 (1990), 34-170.

[13] Meyer, Y., Ondelettes et Opérateurs I: Ondelettes. Hermann, 1990.

[14] Leray, J., Etude de diverses équations intégrales non linéaires et de quelques problèmes que pose l'hydrodynamique. J. Math. Pures et Appl. 12 (1933), 1-82.

[15] Foias, C., Temam, R., Self-similar statistical solutions of the NavierStokes equations. Comm. Math. Phys. 90 (1983), 187-206.

[16] Giga, Y., Miyakawa, T., Navier-Stokes flows in $\mathbb{R}^{3}$ and Morrey spaces. Comm. Partial Diff. Equations. 14 (1989), 577-618.

[17] Jaffard, D., Meyer, Y., Bases d'ondelettes dans des ouverts de $\mathbb{R}^{n}$. J. Math. Pures Appl. 9 (1989), 95-108.

[18] Littlewood, J. E., Paley, R., Theorems on Fourier series and power series II. Proc. London Math. Soc. 42 (1937), 52-89.

Recibido: 8 de abril de 1.996

Marco Cannone

Université de Paris VII

UFR Mathematiques Tour 56-66, 5eme etage 2, Place Jussieu, 75251 Paris Cedex 05

FRANCE

cannone@math. jussieu.fr 